\title{
Revisão sistemática de estudos sobre TPACK na for- mação de professores no Brasil e em Portugal
}

\author{
Priscilla Ramos Lara Ribeiro \\ João Manuel Nunes Piedade \\ Instituto de Educação da Universidade de Lisboa (Portugal)
}

\section{Resumo}

A discussão do quadro Technological Pedagogical Content Knowledge (TPACK) tem sido foco crescente de pesquisas, principalmente entre os formadores de professores, que necessitam preparar os professores para integrar as Tecnologias da Informação e Comunicação (TIC) ao ensino e aprendizagem em sala de aula. Com o objetivo de identificar de que forma o quadro TPACK é utilizado como modelo teórico para o desenho de cursos de formação de professores linicial ou continuada), realizou-se uma revisão sistemática de estudos publicados em contexto brasileiro e português, disponíveis em bases de dados de acesso aberto. Após a aplicação dos critérios de inclusão e exclusão, o corpus de análise constituiu-se de 28 trabalhos publicados entre 2013 e 2020. Os resultados destacam a prevalência em cursos de formação continuada, cursos na área curricular da Matemática e abordagens voltadas para o desenvolvimento do domínio TPK dos professores.

Palavras-chave: Formação de professores. Technological Pedagogical Content Knowledge. Tecnologias na educação. Revisão sistemática.

\section{Teaching of Didactics in the bachelor's degree in pedagogy}

\section{Abstract}

The discussion of the Technological Pedagogical Content Knowledge (TPACK) frame has been a focus of research, particularly among teacher trainers, who need to prepare the teachers to integrate the Information and Communication Technologies (ICT) to the teaching and learning processes in the classroom. Aiming to identify how the TPACK frame is used as a theoretical model to design teacher training programs (both initial and continuing training), a systematic review of the literature has been made using published studies available in open data platforms about the Portuguese and Brazilian contexts. After application of inclusion and exclusion criteria, the corpus for analysis was formed by 28 works, published between 2013 and 2020. Among obtained results stand out: the prevalence of continuing education programs, programs in the mathematics field and programs focusing on developing the teacher's TPK mastery.

Keywords: Teacher training. Technological Pedagogical Content Knowledge. Technologies in education. Systematic review. 


\section{Revisión sistemática de estudios sobre TPACK en la formación del profesorado en Brasil y Portugal}

\section{Resumen}

La discusión del marco Technological Pedagogical Content Knowledge (TPACK), ha sido un foco de investigación, entre los formadores de docentes, que necesitan preparar a los profesores para integrar las Tecnologías de la Información y la Comunicación (TIC) en la enseñanza en el aula. Con el fin de identificar cómo se utiliza el marco TPACK como modelo teórico para el diseño de cursos de formación docente (inicial o continua), se realizó una revisión sistemática de los estudios publicados en el contexto brasileño y portugués, disponibles en bases de datos de acceso abierto. Después de aplicar los criterios de inclusión y exclusión, el corpus de análisis consistió en 28 trabajos, publicados entre 2013 y 2020. Los resultados destacan la prevalencia en los cursos de formación continua, cursos de Matemáticas y los enfoques dirigidos al desarrollo del dominio TPK de los profesores.

Palabras clave: Formación de profesores. Technological Pedagogical Content Knowledge. Tecnologías en educación. Revisión sistemática.

\section{O Modelo Technological Pedagogical Content Knowledge (TPACK)}

Para compreender o quadro teórico TPACK, é necessário revisitar sua origem nas obras do pesquisador Lee Shulman, uma das principais referências nos estudos sobre a profissão docente. Inicialmente intitulado PCK - Conhecimento Pedagógico do Conteúdo lem inglês, Pedagogical Content Knowledge), o conceito foi descrito pela primeira vez pelo autor em 1984 (BORN; PRADO; FELLPPE, 2019) como uma intersecção entre o Conhecimento Pedagógico e o Conhecimento do Conteúdo dos professores.

Dentre as suas preocupações quanto à avaliação da atuação docente, estava a ausência de foco no conteúdo ensinado, já que, à época de suas pesquisas, as avaliações tinham como objetivo verificar unicamente a capacidade de ensinar do professor; o conteúdo específico não era considerado. O autor referia-se a essa questão como o "paradigma perdido" ISHULMAN, 1986), pois entendia que a docência, assim como qualquer outra profissão, exigia uma articulação entre o conhecimento específico (conteúdo) e a prática (instrução). Em sua busca por definir as bases de conhecimento da docência, Shulman (1987) criou sete categorias, sendo o Conhecimento Pedagógico do Conteúdo (PCK) a que "[...] certamente mais influenciou o campo educacional" (BORN; PRADO; FELLPEE, 2019, p. 3). 
Diversos autores, desde então, vêm criando novos modelos sobre o PCK e seu desenvolvimento, muitas vezes trazendo divergências entre o modelo inicial proposto por Shulman, e apresentando novas concepções (FERNANDEZ, 2011 1). Um desses novos modelos explicativos foi criado por Mishra e Koehler (2006) e denomina-se Conhecimento Tecnológico Pedagógico do Conteúdo (TPACK). Esse modelo acrescenta o conhecimento tecnológico à proposta de formação de professores de Shulman (1986; 1987), procurando compreender o que os professores precisam saber para integrar adequadamente as tecnologias em suas práticas, e como desenvolver essas habilidades.

O TPACK procura "[...] articular os conhecimentos pedagógicos (PK), os conhecimentos de conteúdo (CK) e os conhecimentos tecnológicos (TK) com as estratégias escolhidas pelos professores na integração de TDIC em suas práticas" (SANTOS NETO; STRUCHINER, 2019, p. 220).

As intersecções entre esses três conjuntos básicos de conhecimentos (TK, PK e CK) originam quatro domínios diferentes, onde a intersecção central é o conhecimento TPACK, conforme pode ser observado na Figura 1.

\section{Figura 1}

\section{Os domínios do conhecimento TPACK}

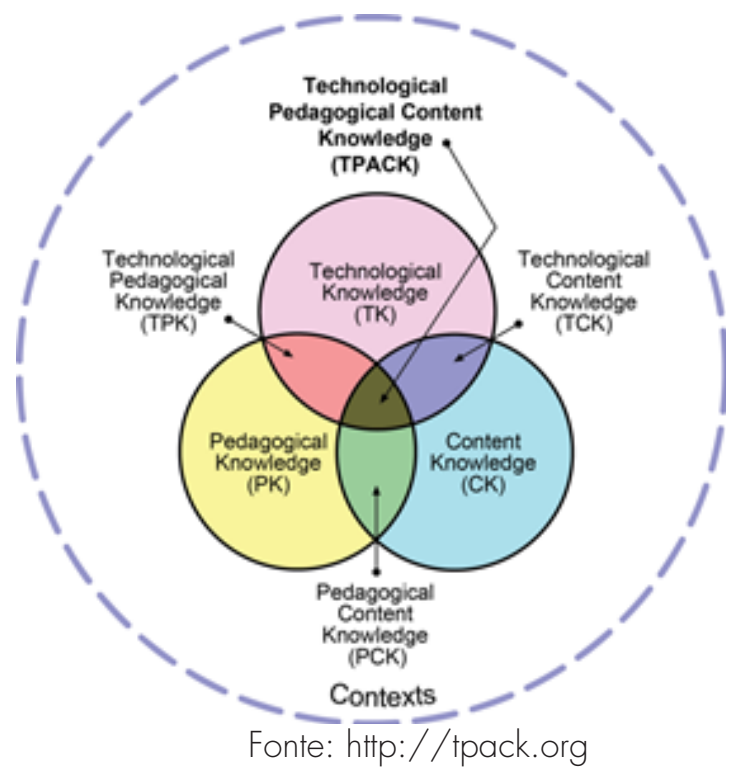


A premissa do Quadro teórico TPACK é, portanto, o equilíbrio dinâmico entre os três elementos-chave (conteúdo, pedagogia e tecnologia) no conjunto de conhecimento dos professores para o fim de ensinar, resultando na integração significativa destes elementos na prática docente.

Segundo Mishra e Koehler (2006), existem diversos programas de formação e workshops que oferecem métodos tradicionais de treinamento em TIC para professores e que são inadequados para um bom ensino com tecnologias, pois: 1) abordam o uso de softwares genéricos; 2) não consideram o estilo de ensino de cada professor e a disciplina específica; 3) não exploram todo o potencial das ferramentas digitais; 4) não consideram a desatualização das tecnologias ao longo do tempo; e 5) não fornecem experiências sobre como aplicá-las em aula.

Ao contrário de um especialista em tecnologias, espera-se que o professor se sinta confortável na utilização das ferramentas disponíveis para, então, desenvolver a análise crítica e a proposição de práticas inovadoras em suas aulas (ROSÁRIO; MOREIRA, 2015), habilidades não contempladas naquele modelo superado de formação de professores.

A relativa novidade das tecnologias educacionais online traz, para os professores, a problemática de lidarem com os três fatores /conteúdo, pedagogia e tecnologia) e as relações entre eles, levando-os a fazer perguntas sobre sua própria pedagogia, algo que podem não ter feito há muito tempo (PERUSKI; MISHRA, 2004). Neste contexto, a discussão do modelo TPACK tem sido um foco crescente de pesquisas, principalmente entre os formadores de professores, que necessitam preparar os professores para integrar as $\mathrm{TIC}$ ao ensino e aprendizagem em sala de aula (CHAl; $\mathrm{KOH}$; TSAl, 2013).

\section{Duas propostas para desenvolvimento do TPACK na formação inicial e continuada de professores}

A fim de consolidar referências sobre o TPACK acerca de sua teoria, práticas e pesquisas em diferentes áreas do conhecimento e na formação de professores, Herring, Koehler e Mishra (2008) editaram o livro Handbook of technological pedagogical content knowledge for educators, o qual classificaram como "[...] a mandate and a manifesto on the engagement of technology in classrooms". Em sua última edição (2016), os capítulos 11 e 12 da seção III apresentam, respectivamente, modelos de formação inicial e de formação 
continuada para promoção do TPACK junto aos futuros professores e aos já em exercício.

No capítulo 11, Mouza (2016) trata do desenvolvimento do TPACK pelos professores em formação inicial e busca sintetizar as principais pesquisas realizadas na área desde a primeira edição do livro, em 2008. Como propostas para o desenvolvimento do TPACK na formação inicial, a autora sugere três modelos de curso, representados na Tabela 1.

\section{Tabela 1}

\section{Propostas de desenvolvimento do TPACK na formação inicial}

\begin{tabular}{|l|}
\hline Proposta de Curso \\
\hline 1) Curso autônomo de tecnologia educacional: curso básico de conhecimento \\
tecnológico (TK) oferecido aos futuros professores e isolado das demais disciplinas \\
de sua graduação, com o objetivo de promover habilidades e confiança para o \\
uso das tecnologias em sala de aula. \\
\hline 2) Estratégias de formação inseridas dentro de uma disciplina de educação tec- \\
nológica ou dentro de uma disciplina de didática ou metodologia: este modelo \\
apresenta quatro abordagens: Design instrucional; Desenvolvimento de caso base- \\
ado em TPACK; Reflexão e Experiência de campo. \\
\hline 3) Estratégias instrucionais implementadas durante todo o programa de formação \\
de professores: pesquisa recente aponta que há um substancial ganho de quali-- \\
dade dos domínios TPACK com a utilização destas estratégias; porém, a cada \\
ano de formação, estes conhecimentos costumam perder-se, tornando necessário \\
o espaçamento da instrução e retomada dos conceitos até o término do curso \\
(MOUZA; NANDAKUMAR; OZDEN; KARCHMER; KLEIN, 2017).
\end{tabular}

Adaptado de Mouza (2016)

capítulo 12, escrito por Harris (2016), trata especificamente sobre as tendências, modelos e trajetórias para o desenvolvimento do TPACK na formação continuada de professores. Esse capítulo apresenta oito abordagens sobre como promover o conhecimento TPACK na formação continuada, que serão detalhadas na Tabela 2. 
Revisão sistemática de estudos sobre TPACK na formação de professores no Brasil e em Portugal

\section{Tabela 2}

\section{Abordagens de desenvolvimento do TPACK na formação continuada}

\begin{tabular}{l} 
Abordagens para o desenvolvimento TPACK \\
\hline 1) Design instrucional colaborativo: desenho de um projeto educacional, unidade \\
curricular ou curso que será depois testado por um pequeno grupo de professores \\
com diferentes formações e conhecimentos sobre tecnologia educacional. \\
\hline 2) Método focado em PCK: análise de práticas docentes que recorram à utilização \\
pedagógica de ferramentas digitais no contexto específico das áreas curriculares. \\
\hline 3) Método focado em TPK: análise e avaliação das potencialidades pedagógicas \\
das ferramentas digitais, observando-se a sua possível utilização em sala de aula \\
para potencializar o trabalho de professores e alunos, seus pontos fortes e limita- \\
ções, e a sua adequação a determinada área curricular. \\
\hline 4) Método reflexivo: diferente dos anteriores, tem um foco mais individual sobre \\
cada professor e seu contexto, buscando identificar quais são as suas necessidades \\
de aprendizagem dentro dos domínios TPACK, bem como os avanços decorrentes \\
da instrução do método. \\
\hline 5) Métodos baseados em problemas: os professores são divididos em pequenos \\
grupos para discussão de problemas que encontram em suas práticas diárias em \\
sala de aula; juntos, selecionam alguns para discussão de soluções, implementam- \\
-nas e refletem sobre os seus resultados. Esse processo é todo compartilhado com os \\
demais integrantes do curso de formação. \\
\hline b) Métodos de aprendizagem adaptativa: semelhantes ao método reflexivo, buscam \\
criar perfis TPACK individualizados dos professores e recomendações para melhoria \\
contínua das suas habilidades. A diferença consiste na utilização de instrumentos \\
de recolha de dados desenhados especificamente para sistematizar essas informa- \\
ções, gerando um diagnóstico sobre suas principais dificuldades e os caminhos \\
para superá-las.
\end{tabular}




\section{Tabela 2}

Abordagens de desenvolvimento do TPACK na formação continuada (continuação)

8) Métodos de aprendizagem no local de trabalho: assim como os métodos de planejamento instrucional, ocorrem durante a prática de trabalho diária do professor. Nesses métodos, sugere-se a constituição de comunidades de prática com seus alunos e demais membros envolvidos no contexto educacional, conjuntamente. Tal comunidade permitirá a troca de informações e experiências, bem como a negociação de sentidos, os quais tornam estes métodos bastante complexos, mas enriquecedores para o desenvolvimento global do TPACK.

Adaptado de Harris (2016).

Na revisão sistemática de literatura que se apresenta a seguir, usaremos os modelos e estratégias definidas por Mouza (2016) e Harris (2016) para analisar a forma como o modelo TPACK vem sendo utilizado na formação inicial e continuada de professores.

\section{Métodos}

Com a finalidade de compreender como o quadro TPACK é utilizado como base teórica nos programas de formação inicial e continuada de professores das diversas áreas curriculares, realizou-se uma revisão sistemática da literatura (GOUGH; THOMAS; OLIVER, 2012) sobre estudos publicados em contexto brasileiro e português, disponíveis em bases de dados de acesso aberto. Nesse âmbito, recorremos ao protocolo de pesquisa definido por Kitchenham (2004), que apresenta as seguintes fases: (1) definição das questões de pesquisa; (2) seleção das fontes e critérios de pesquisa; (3) definição dos critérios de inclusão e exclusão; (4) extração e sintetização dos resultados; e (5) produção de conclusões.

\section{Questões de Pesquisa}

De modo a corresponder ao objetivo definido para a busca de estudos e evidências sobre a utilização do modelo TPACK na formação do professor, foram definidas as seguintes questões de pesquisa: (1) Existem trabalhos 
publicados, no Brasil ou em Portugal sobre o modelo TPACK na formação de professores? (2) Qual é a tipologia dos estudos sobre o modelo TPACK na formação de professores? (3) Quais são as abordagens do modelo TPACK na concepção dos cursos de formação inicial e continuada de professores? (4) $\bigcirc$ modelo TPACK é mais utilizado em cursos de formação inicial ou continuada de professores? (5) Quais são as áreas curriculares de formação de professores que mais recorrem ao modelo TPACK? (6) Qual é a utilização do modelo TPACK nos cursos de formação de professores para atuarem em diferentes modalidades de ensino (EaD, Híbrido e Presencial)? (7) Que potencialidades e limitações emergem nos estudos sobre a utilização do modelo TPACK na formação de professores?

\section{Fontes e critérios de pesquisa}

Para desenvolver a pesquisa, foram selecionadas cinco bases de dados de acesso aberto que disponibilizam trabalhos publicados de ambos os países: (i) Catálogo de Teses e Dissertações da CAPES (Coordenação de Aperfeiçoamento de Pessoal de Nível Superior); (ii) Directory of Open Access Journals - DOAj; (iii) Periódicos CAPES; (iv) Repositórios Científicos de Acesso Aberto de Portugal - RCAAP; e (v) Scientific Electronic Library Online - SciELO. Os termos de busca utilizados foram: "TPACK"; "CTPC" |conhecimento tecnológico pedagógico do conteúdo); "formação de professores" e "desenvolvimento profissional". Tais termos foram combinados em equações de pesquisa com o auxílio do operador booleano "E" nos campos de busca de todas as bases de dados: "TPACK" E "formação de professores"; "CTPC" E "formação de professores"; "TPACK" E "desenvolvimento profissional"; "CTPC" E "desenvolvimento profissional".

A coleta dos dados ocorreu durante todo o mês de janeiro de 2021 e foram utilizados como critérios de inclusão: (i) trabalhos científicos empíricos, em formato de artigo, dissertação de mestrado ou tese de doutoramento; (ii) realizados no Brasil ou Portugal; (iii) publicados em língua portuguesa; (iv) de acesso livre; e (v) publicados entre 2006, ano no qual foi definido o modelo TPACK, e 2020. A pesquisa devolveu um total de 123 trabalhos, distribuídos de acordo com a Tabela 3. Os trabalhos encontrados foram organizados e sistematizados em uma folha de cálculo na plataforma Google Sheets, contendo: Título, Autor, Palavras-chave, Resumo, Formato da publicação, Ano, Local do 
estudo, Contexto do Estudo, Área, Tipo de Formação, Abordagem TPACK, Ligação de Acesso e Base de Dados.

\section{Tabela 3}

Resultado das buscas em cinco bases de dados

\begin{tabular}{|l|c|c|}
\hline \multicolumn{1}{|c|}{ Bases de Dados } & $\begin{array}{c}\text { P u b l i c a ç õ e s } \\
\text { encontradas }\end{array}$ & $\begin{array}{c}\text { Pe rí o do do d a s } \\
\text { publicações }\end{array}$ \\
\hline $\begin{array}{l}\text { Catálogo de Teses e Dissertações da } \\
\text { CAPES }\end{array}$ & 70 & $2012-2019$ \\
\hline DOAJ & 5 & $2016-2019$ \\
\hline Periódicos CAPES & 13 & $2010-2020$ \\
\hline RCAAP & 31 & $2011-2019$ \\
\hline SciELO & 4 & $2015-2020$ \\
\hline Total & 123 & $2010-2020$ \\
\hline
\end{tabular}

Fonte: pesquisa empírica dos autores.

Após a análise cruzada dos resultados das diferentes bases de dados, foram eliminadas 21 publicações repetidas. $O$ passo seguinte consistiu na leitura dos resumos das publicações, a fim de se analisar se estavam dentro do escopo do trabalho, ou seja, se tratavam de cursos/dinâmicas de formação de professores que utilizaram o quadro TPACK como base teórica para sua elaboração e desenvolvimento, e se cumpriam os critérios de inclusão. Da leitura, foram excluídos 74 trabalhos em que o quadro TPACK se apresentou apenas como ferramenta para análise dos resultados encontrados nas publicações, ou relacionados a cursos ou disciplinas baseadas em TPACK, mas voltados para alunos do ciclo básico, ensino médio ou cursos de graduação 
Revisão sistemática de estudos sobre TPACK na formação de professores no Brasil e em Portugal

não relacionados à formação de professores. Assim, o corpus final de análise é constituído por 28 publicações, representados e categorizados na Tabela 4.

\section{Tabela 4}

\section{Lista de publicações selecionadas para análise}

\begin{tabular}{|c|c|c|c|}
\hline \multicolumn{4}{|c|}{ Periódico CAPES } \\
\hline Cód. & Autores & Título & Tipo \\
\hline Al 1 & $\begin{array}{l}\text { Alcântara; Dullius; } \\
\text { Carreira (2016) }\end{array}$ & $\begin{array}{l}\text { O desenvolvimento do professor: uma } \\
\text { proposta de formação continuada cen- } \\
\text { trada nas tecnologias e ancorada na } \\
\text { prática }\end{array}$ & Artigo \\
\hline A2 & Cyrino (2017) & $\begin{array}{c}\text { Ações da formadora e a dinâmica de } \\
\text { uma comunidade de prática na consti- } \\
\text { tuição/mobilização de TPACK }\end{array}$ & Artigo \\
\hline A3 & $\begin{array}{l}\text { Oliveira; Henriques; } \\
\text { Gutiérrez-Fallas } \\
\text { (2018) }\end{array}$ & $\begin{array}{l}\text { A integração da tecnologia na plani- } \\
\text { ficação de aulas na perspectiva do } \\
\text { ensino exploratório: um estudo com fu- } \\
\text { turos professores de matemática }\end{array}$ & Artigo \\
\hline A4 & $\begin{array}{c}\text { Sousa; Terçariol; } \\
\text { Gitahy (2017) }\end{array}$ & $\begin{array}{c}\text { Conhecimento Tecnológico Pedagógico } \\
\text { do Conteúdo: construção de conceitos } \\
\text { e habilidades didáticas }\end{array}$ & Artigo \\
\hline A5 & Sampaio (2016) & $\begin{array}{c}\text { Desenvolvimento profissional dos profes- } \\
\text { sores de Matemática: uma experiência } \\
\text { de formação em TIC }\end{array}$ & Artigo \\
\hline$A b$ & $\begin{array}{l}\text { Gutierrez-Fallas; } \\
\text { Henriques (2020) }\end{array}$ & $\begin{array}{l}\text { TPACK de futuros professores de } \\
\text { Matemática numa experiência de } \\
\text { formação }\end{array}$ & Artigo \\
\hline$A 7$ & Sampaio (2015) & $\begin{array}{l}\text { Integração de Quadros Interativos no } \\
\text { Ensino da Matemática - desenvolvimen- } \\
\text { to Profissional de Professores }\end{array}$ & Artigo \\
\hline
\end{tabular}




\section{Tabela 4}

Lista de publicações selecionadas para análise (continuação)

\begin{tabular}{|c|c|c|c|}
\hline \multicolumn{4}{|c|}{ Catálogo de Teses e Dissertações da CAPES } \\
\hline Cód. & Autores & Título & Tipo \\
\hline A8 & Martins (2016) & $\begin{array}{l}\text { O potencial de Fóruns de discussão em } \\
\text { Comunidades Virtuais de Aprendizagem } \\
\text { como ferramenta de colaboração entre } \\
\text { licenciados e professores de Biologia. }\end{array}$ & $\begin{array}{l}\text { Dissertação de } \\
\text { Mestrado }\end{array}$ \\
\hline A9 & Lotthammer (2019) & $\begin{array}{l}\text { Proposta de modelo de plano de aula } \\
\text { para auxiliar docentes na elaboração } \\
\text { de aulas mediadas pelas tecnologias } \\
\text { da informação e comunicação (TIC) }\end{array}$ & $\begin{array}{l}\text { Dissertação de } \\
\text { Mestrado }\end{array}$ \\
\hline AlO & Alcântara (2015) & $\begin{array}{l}\text { A trajetória de desenvolvimento do pro- } \\
\text { fessor na utilização de tecnologias nas } \\
\text { aulas de Matemática em um contexto de } \\
\text { formação continuada }\end{array}$ & $\begin{array}{l}\text { Dissertação de } \\
\text { Mestrado }\end{array}$ \\
\hline A11 & Bervian (2019) & $\begin{array}{l}\text { Processo de Investigação-Formação- } \\
\text { Ação Docente: uma perspectiva } \\
\text { de constituição do Conhecimento } \\
\text { Tecnológico Pedagógico do Conteúdo }\end{array}$ & $\begin{array}{l}\text { Tese de } \\
\text { Doutoramento }\end{array}$ \\
\hline $\mathrm{A} 12$ & Oliveira (2018) & $\begin{array}{l}\text { Percepções de professores sobre a } \\
\text { TPACK no Ensino Superior }\end{array}$ & $\begin{array}{l}\text { Dissertação de } \\
\text { Mestrado }\end{array}$ \\
\hline A 13 & Oliveira (2017) & $\begin{array}{l}\text { Tecnologias Digitais na formação conti- } \\
\text { nuada: situações de ensino articulando } \\
\text { geometria e funções }\end{array}$ & $\begin{array}{l}\text { Dissertação de } \\
\text { Mestrado }\end{array}$ \\
\hline Al4 & Schroder (2018) & $\begin{array}{l}\text { Tecnologias móveis: desafios e pers- } \\
\text { pectivas no ensino e aprendizagem de } \\
\text { Matemática }\end{array}$ & $\begin{array}{l}\text { Dissertação de } \\
\text { Mestrado }\end{array}$ \\
\hline A 15 & Silva (2018) & $\begin{array}{c}\text { Formação continuada: um estudo sobre } \\
\text { integração de tecnologia digital para } \\
\text { ensinar poliedros }\end{array}$ & $\begin{array}{c}\text { Tese } \quad d e \\
\text { Doutoramento }\end{array}$ \\
\hline A16 & Rabello (2015) & $\begin{array}{c}\text { Tecnologias digitais e ensino superior: } \\
\text { uma experiência de desenvolvimento } \\
\text { profissional docente na UFRJ }\end{array}$ & $\begin{array}{c}\text { Tese } \quad d e \\
\text { Doutoramento }\end{array}$ \\
\hline
\end{tabular}


Revisão sistemática de estudos sobre TPACK na formação de professores no Brasil e em Portugal

\section{Tabela 4}

Lista de publicações selecionadas para análise (continuação)

\begin{tabular}{|c|c|c|c|}
\hline Cód. & Autores & Título & Tipo \\
\hline A16 & Rabello (2015) & $\begin{array}{l}\text { Tecnologias digitais e ensino superior: } \\
\text { uma experiência de desenvolvimento } \\
\text { profissional docente na UFRJ }\end{array}$ & $\begin{array}{c}\text { Tese de } \\
\text { Doutoramento }\end{array}$ \\
\hline A 17 & Vieira (2013) & $\begin{array}{l}\text { Grupo de estudos de professores e a } \\
\text { apropriação de tecnologia digital no } \\
\text { ensino de geometria: caminhos para o } \\
\text { conhecimento profissional }\end{array}$ & $\begin{array}{c}\text { Tese de } \\
\text { Doutoramento }\end{array}$ \\
\hline A18 & Porto (2016) & $\begin{array}{l}\text { Formação continuada do professor de } \\
\text { Matemática para uso do GeoGebra em } \\
\text { dispositivo mobile }\end{array}$ & $\begin{array}{c}\text { Tese de } \\
\text { Doutoramento }\end{array}$ \\
\hline Al9 & Rocha (2015) & $\begin{array}{l}\text { A programação de computadores } \\
\text { como meio para integrar diferentes } \\
\text { conhecimentos: uma experiência com } \\
\text { professores de Matemática }\end{array}$ & $\begin{array}{l}\text { Tese de } \\
\text { Doutoramento }\end{array}$ \\
\hline A20 & Padilha (2015) & $\begin{array}{l}\text { Apropriação das tecnologias digitais } \\
\text { móveis para explorar funções polino- } \\
\text { miais do } 1^{\circ} \text { grau }\end{array}$ & $\begin{array}{c}\text { Dissertação de } \\
\text { Mestrado }\end{array}$ \\
\hline A2 1 & Baldini (2014) & $\begin{array}{l}\text { Elementos de uma Comunidade de } \\
\text { Prática que permitem o desenvolvimento } \\
\text { profissional de professores e futuros pro- } \\
\text { fessores de Matemática na utilização } \\
\text { do Software GeoGebra. }\end{array}$ & $\begin{array}{l}\text { Tese de } \\
\text { Doutoramento }\end{array}$ \\
\hline \multicolumn{4}{|c|}{ Repositórios Científicos de Acesso Aberto de Portugal - RCAAP } \\
\hline A22 & Rocha (2015) & $\begin{array}{l}\text { O conhecimento tecnológico e pedagó- } \\
\text { gico do conteúdo (TPACK) aplicado ao } \\
\text { ensino de Geografia }\end{array}$ & $\begin{array}{c}\text { Tese de } \\
\text { Doutoramento }\end{array}$ \\
\hline A23 & Leite (2017) & $\begin{array}{l}\text { Formação de professores de } \\
\text { Matemática e tecnologias digitais: um } \\
\text { estudo sobre o Teorema de Tales }\end{array}$ & $\begin{array}{c}\text { Dissertação de } \\
\text { Mestrado }\end{array}$ \\
\hline
\end{tabular}




\section{Tabela 4}

Lista de publicações selecionadas para análise (continuação)

\begin{tabular}{|c|c|c|c|}
\hline Cód. & Autores & Tí́tulo & Tipo \\
\hline A24 & Esquincalha (2015) & $\begin{array}{l}\text { Conhecimentos revelados por tułores em } \\
\text { um curso de formação continuada para } \\
\text { professores de Matemática na modali- } \\
\text { dade a distância }\end{array}$ & $\begin{array}{l}\text { Tese de } \\
\text { Doutoramento }\end{array}$ \\
\hline A25 & Moreira (2014) & $\begin{array}{c}\text { O Facebook e a formação contínua de } \\
\text { educadores de infância e professores } \\
\text { do } 1^{\circ} \text { ciclo do ensino básico: da forma- } \\
\text { ção à integração das TIC }\end{array}$ & $\begin{array}{c}\text { Dissertação de } \\
\text { Mestrado }\end{array}$ \\
\hline A26 & Barbosa (2014) & $\begin{array}{l}\text { Repensar as TIC nas práticas letivas - da } \\
\text { formação à integração: um estudo com } \\
\text { educadores de infância e professores } \\
\text { do } 1^{\circ} \text { ciclo do ensino básico } \\
\end{array}$ & $\begin{array}{l}\text { Dissertação de } \\
\text { Mestrado }\end{array}$ \\
\hline A27 & Cibotto (2015) & $\begin{array}{l}\text { O uso pedagógico das tecnologias da } \\
\text { informação e comunicação na forma- } \\
\text { ção de professores: uma experiência na } \\
\text { licenciatura em Matemática }\end{array}$ & $\begin{array}{c}\text { Tese de } \\
\text { Doutoramento }\end{array}$ \\
\hline A28 & Nakashima (2014) & $\begin{array}{l}\text { A dialética dos conhecimentos peda- } \\
\text { gógicos dos conteúdos tecnológicos e } \\
\text { suas contribuições para a ação docente } \\
\text { e para o processo de aprendizagem } \\
\text { apoiados por um ambiente virtual }\end{array}$ & $\begin{array}{c}\text { Tese de } \\
\text { Doutoramento }\end{array}$ \\
\hline
\end{tabular}

Fonte: pesquisa empírica dos autores.

\section{Resultados}

Ao analisarmos o número de publicações por ano, observa-se que os primeiros trabalhos sobre o TPACK, no contexto desta pesquisa, começaram a ser publicados a partir do ano de 2013, com ascensão até o ano de 2015. 


\section{Gráfico 1}

Número de trabalhos selecionados por ano de publicação

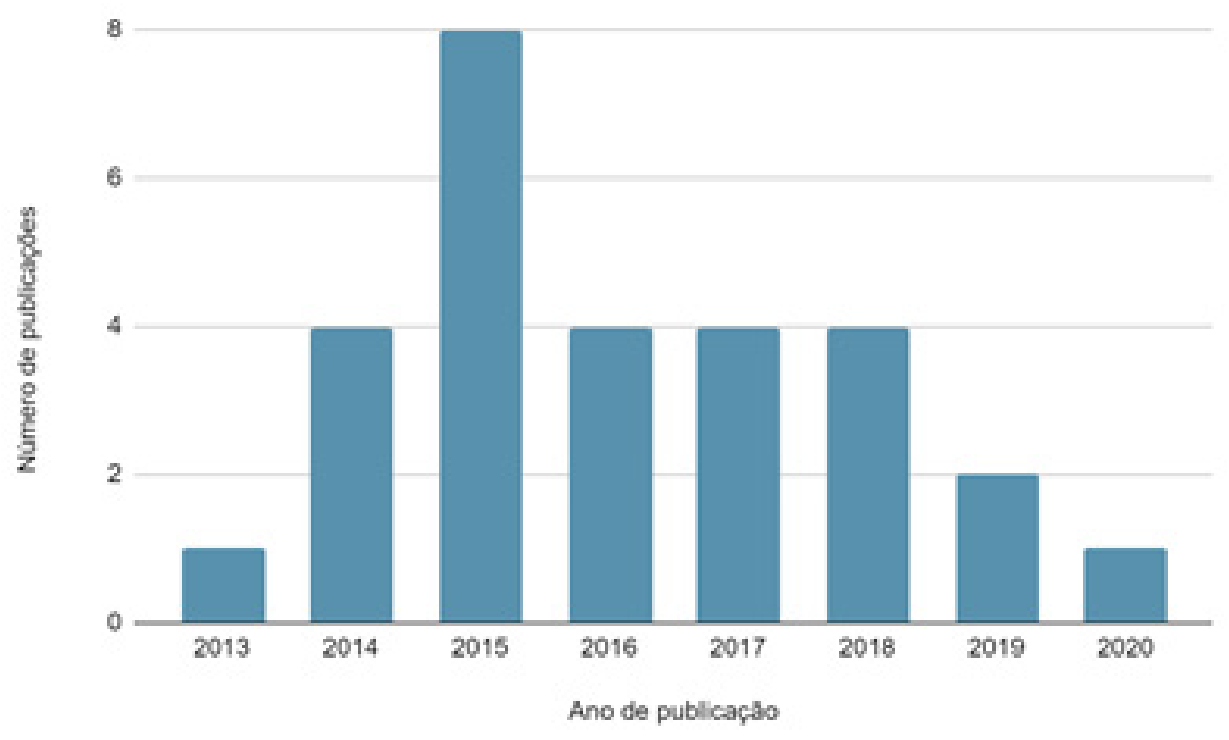

Fonte: pesquisa empírica dos autores.

Esse resultado é coincidente com o estudo internacional de Soler-Costa, Moreno-Guerrero, López-Belmonte e Marín-Marín (2021), que desenvolveram um estudo bibliométrico do termo TPACK em publicações da base de dados Web of Science (WoS). Neste estudo, verificou-se que, no período compreendido entre 2006 e 2014 , o tema com segundo maior desempenho acadêmico foi "formação de professores". Entre 2015 e 2017, os temas do período anterior permaneceram os mesmos; contudo, foram a eles acrescidas novas nuances, como "integração tecnológica" e "respeito", ocasionando uma ligeira queda nas publicações sobre formação de professores. Já em 2018 e 2019, há uma mudança de tendência e novas linhas de investigação começam a surgir, com foco na validação de instrumentos de avaliação do TPACK e na percepção dos professores sobre esse quadro teórico, o que é demonstrado no Gráfico 1 por uma reta descendente quanto ao tema da formação de professores. Em relação ao local de estudo, foram encontrados 21 trabalhos no Brasil, 6 em Portugal e 1 que utilizou dados de ambos os países. 


\section{Gráfico 2 \\ Distribuição por países com relação aos tipos de publicação}

\section{Tipo de publicação}

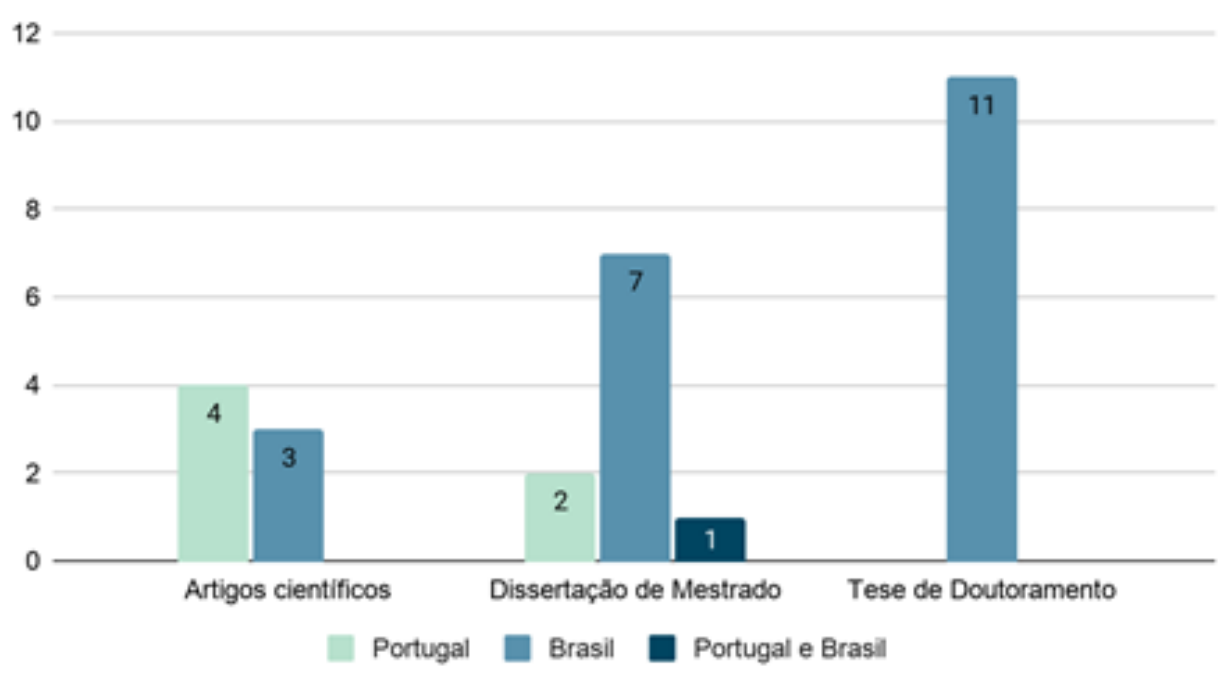

Fonte: pesquisa empírica dos autores.

Gráfico 2 demonstra que, apesar do número elevado de dissertações de mestrado e teses de doutoramento realizadas no Brasil, o número de publicações de artigos científicos na área discutida é maior em Portugal. Destaca-se também o fato de não haver, em Portugal, teses de doutoramento sobre o tema nas bases de dados pesquisadas.

Quanto à abordagem do modelo TPACK nos cursos de formação, foi realizada uma classificação prévia das publicações, utilizando-se como referencial as duas propostas para o desenvolvimento do TPACK na formação inicial e continuada, apresentadas anteriormente.

Relativamente à utilização do modelo TPACK na formação inicial e continuada de professores, foi possível encontrar as evidências apontadas no Gráfico 3. 


\section{Gráfico 3 \\ Trabalhos científicos por tipo de formação}

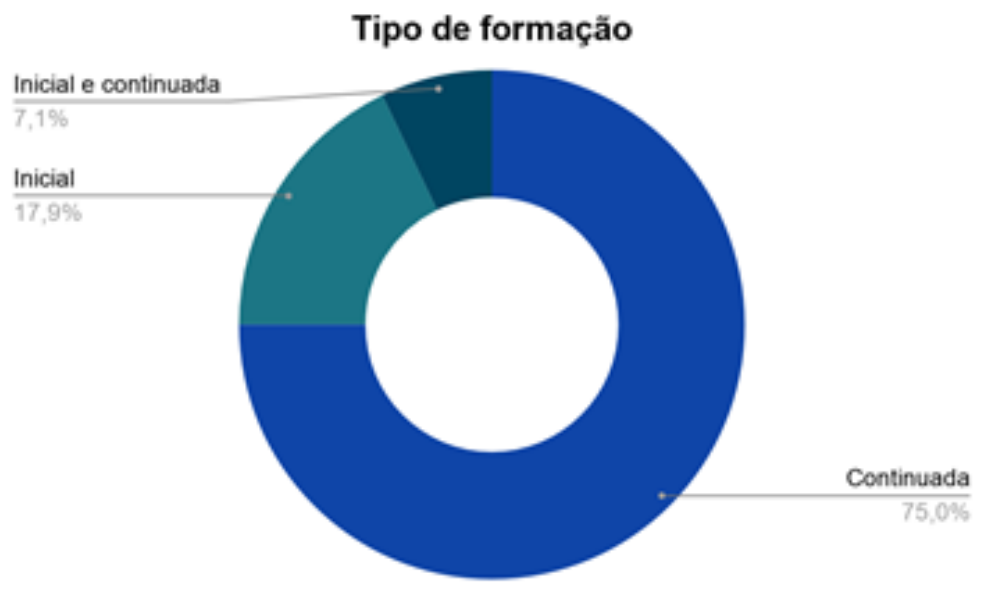

Fonte: pesquisa empírica dos autores.

16 Considerando os tipos de formação de professores, as publicações selecionadas contemplaram, em sua grande maioria, programas de Formação Continuada. Uma pequena parcela de 7,1\% das publicações apresenta programas em duas categorias, Inicial e Continuada, pois, nesses estudos, licenciandos e professores em exercício interagiram e participaram das mesmas atividades de formação.

Quanto às áreas científicas de incidência das publicações, mais da metade são da Matemática (64,3\%), seguida por Docência no Ensino Superior, Pós-graduação e Pedagogia, com 7,1\% cada e Ciências, Biologia, Geografia e Química com 3,6\% cada. Dentre as publicações da área da Matemática, foram utilizados nos programas de formação os seguintes softwares: TinkerPlotsTM (T3), GeoGebra, Excel, Winplot, Mentimeter, Socrative, Padlet, Grapher, SketchUp, Construfig3D e Régua e Compasso, sendo o software GeoGebra o mais citado, em 66,6\% das publicações.

Utilizando-se a classificação de Mouza (2016) para a formação inicial de professores, as cinco publicações desta categoria foram classificadas de acordo com a tabela 5. 


\section{Tabela 5}

\section{Abordagem TPACK na Formação Inicial, dividida por país}

\begin{tabular}{|l|c|c|}
\hline \multicolumn{1}{|c|}{ Abordagem TPACK } & Brasil & Portugal \\
\hline $\begin{array}{l}\text { Estratégias de formação inseridas } \\
\text { dentro de uma disciplina }\end{array}$ & 2 & 2 \\
\hline $\begin{array}{l}\text { Curso autônomo de tecnologia } \\
\text { educacional }\end{array}$ & 1 & 0 \\
\hline
\end{tabular}

Fonte: pesquisa empírica dos autores.

As opções mais adotadas, em ambos os países, são as estratégias de abordagem do TPACK dentro de uma disciplina da matriz curricular dos licenciandos, geralmente as de Educação Tecnológica, Didática ou Metodologia. Nota-se que a abordagem de estratégias de formação durante toda a graduação dos licenciandos não foi encontrada nas publicações selecionadas.

Com relação à abordagem TPACK em Programas de Formação Continuada e utilizando-se a classificação de Harris (2016), as publicações foram categorizadas da seguinte forma:

\section{Gráfico 4}

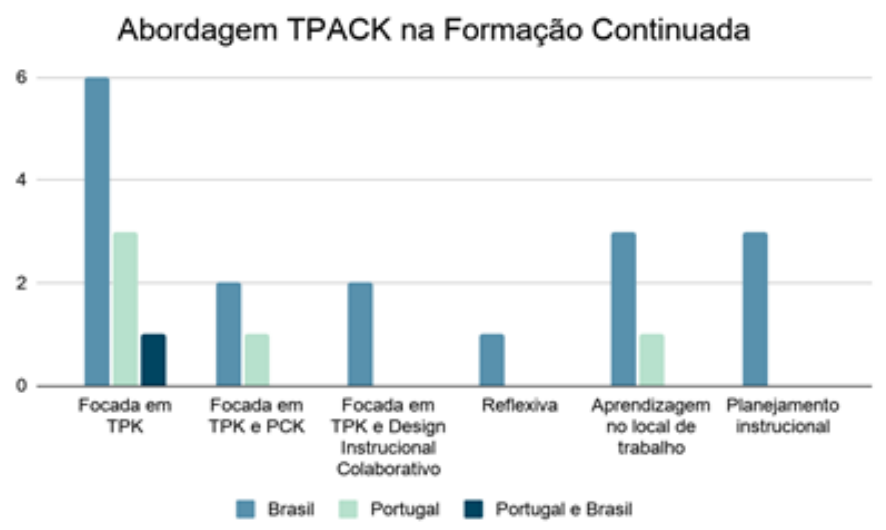

Fonte: pesquisa empírica dos autores. 
Tanto em Portugal como no Brasil, a abordagem mais utilizada é o método focado em TPK (Conhecimento Pedagógico Tecnológico), que consiste na análise de uma ferramenta tecnológica específica, com indicações e discussões sobre suas funcionalidades e potencialidades em relação ao processo de aprendizagem dos alunos. Os métodos baseados em problemas e de aprendizagem adaptativa não foram encontrados nas publicações selecionadas.

Além das abordagens exclusivamente focadas em TPK, encontram-se combinações de TPK com o método PCK /Conhecimento Pedagógico do Conteúdo) e com o Design Instrucional Colaborativo, reforçando a preferência do método TPK nos estudos selecionados, parcialmente ou em sua totalidade.

\section{Discussão dos Resultados}

Para concluir esta revisão, serão novamente apresentadas as perguntas de pesquisa com suas respectivas respostas, com base nos resultados encontrados:

1) Existem trabalhos publicados no Brasil e em Portugal sobre o modelo TPACK na formação de professores? Os primeiros trabalhos nessa área foram publicados a partir de 2013 no Brasil e 2014 em Portugal. Considerando-se a publicação do modelo teórico de Mishra e Koehler no ano de 2006, houve um espaço temporal de sete anos (2006-2013) para o início dos trabalhos empíricos sobre TPACK e formação de professores no Brasil e em Portugal. Já nos Estados Unidos e na Turquia, os dois países com maior volume de produção científica sobre o tema TPACK até o momento (SOLER-COSTA; MORENO-GUERRERO; LÓPEZ-BELMONTE; MARÍN-MARÍN, 20211), observou-se em 2013 o primeiro pico de publicações na área, sendo a linha de pesquisa "formação de professores" a segunda mais estudada.

2) Qual é a tipologia dos estudos sobre o modelo TPACK na formação de professores? Dentre os tipos de estudos selecionados como critérios de inclusão, Portugal apresenta o maior número de artigos científicos, e o Brasil o maior número de teses de doutoramento. Não foram encontradas teses de doutoramento em Portugal sobre esta temática.

3) Quais são as abordagens do modelo TPACK na concepção destes cursos? As abordagens do modelo na formação inicial de professores contemplaram, em sua maioria, estratégias TPACK dentro de disciplinas da matriz curricular dos licenciandos, como Educação Tecnológica, Didática ou 
Metodologia. A incorporação de questões sobre o ensino de tecnologias em disciplinas pedagógicas tem se tornando uma tendência, dado que as mudanças tecnológicas ocorrem em ritmo acelerado e essas disciplinas estruturam toda a formação inicial de professores (OLIVEIRA; HENRIQUES; GUTIÉRREZ-FALLAS, 2018 ). Apenas um dos trabalhos [A23] se utilizou de um curso autônomo sobre tecnologias.

As abordagens do modelo na formação continuada de professores utilizaram, sobretudo, métodos focados em TPK, onde, primeiro, a ferramenta tecnológica é proposta para, então, os professores criarem estratégias para integrá-la em sua prática. Na revisão de literatura de autoria de Hofer e Harris (2012) são citadas experiências de desenvolvimento profissional de professores e observou-se a ênfase em TPK em detrimento aos demais subdomínios do modelo TPACK. Os autores também destacaram a característica amplamente tecnocêntrica destas formações, com foco em tecnologias sem aplicação específica para os conteúdos, e o desempenho menor de TCK (Conhecimento Tecnológico do Conteúdo) no conhecimento TPACK dos professores participantes.

4) O TPACK é mais utilizado em cursos de formação inicial ou continuada de professores? Os cursos de formação continuada foram encontrados em $75 \%$ das publicações selecionadas. Resultado semelhante também pode ser observado na revisão sistemática de Bernardes e Andrade Neto (2020) para a área de Química, na qual se detectou a prevalência de 70\% dos artigos selecionados voltados para formação continuada de professores.

No presente trabalho, $76,2 \%$ das publicações que utilizaram a formação continuada têm o Brasil como local de estudo. Segundo Almeida (2008), é comum encontrar a disciplina de Tecnologia Educativa no currículo das formações de professores em Portugal, tanto iniciais quanto continuadas. Já no Brasil, primeiro surgiram especializações e linhas de pesquisa no mestrado e no doutoramento (formação continuada), para, então, o ensino das TIC começar a ser contemplado nos currículos dos cursos de formação inicial, o que explicaria os valores mais baixos nesta modalidade de formação.

5) Quais são as áreas curriculares de formação de professores que mais recorrem ao modelo TPACK? A área curricular mais recorrente foi a da Matemática, com 64,3\% de incidência. A prevalência da área da Matemática em estudos sobre o TPACK também é apontada nas revisões sistemáticas de 
Revisão sistemática de estudos sobre TPACK na formação de professores no Brasil e em Portugal

Rolando, Luz e Salvador (20 15), Nogueira, Pessoa e Gallego (2015) e Marinho (2018), indicando uma tendência de utilização do quadro teórico TPACK nesta área científica. Verifica-se também a necessidade de mais estudos na área de ciências humanas, a qual apresenta um campo aberto e inédito para propostas de formação de professores com o uso de tecnologias (MARINHO, 2018).

6) Qual é a utilização do modelo TPACK nos cursos de formação de professores para atuarem em diferentes modalidades de ensino (EaD, Hibrido e Presencial)? Foi encontrado apenas um trabalho científico sobre a formação de professores para a modalidade do Ensino a Distância [A24], com foco na atuação dos tutores junto aos alunos da licenciatura em Matemática; os demais contemplaram o ensino presencial. Nesse estudo [A24], foi proposta a expansão do quadro TPACK, com a inclusão do componente afetivo-atitudinal como o quarto conhecimento fundamental para a formação de professores no contexto da Educação a Distância. Intitulado TPACK-OTE IOTE: Online Teacher Education), este modelo considera elementos como a empatia, capacidade para motivar e cordialidade como fundamentais para o estabelecimento de vínculos afetivos com os participantes do curso de formação para EaD e destes com seus alunos.

20 Com relação à inclusão de novos domínios ao quadro teórico TPACK, observa-se que os pesquisadores que estudam a estrutura teórica do modelo costumam discutir as relações entre seus domínios de conhecimento. Segundo Rosenberg e Koehler (2015), alguns pesquisadores os conceituam como integrativos, onde cada domínio é único e considerado distinto dos demais, ou como transformadores, onde cada domínio é indissociável e analisado de forma holística. Também se discute sobre o refinamento destes domínios, com a sugestão de inclusão de mais elementos ou retirada de outros. No caso do trabalho científico [A24], talvez fosse uma opção considerar as especificidades da Educação a Distância como parte do componente "Contexto", poucas vezes analisado nos estudos teóricos sobre o TPACK (ROSENBERG; KOEHLER, 2015), dado que é a partir da análise e correta identificação das suas nuances que os professores podem ajustar suas práticas, permitindo-thes chegar a todo e qualquer aluno (BARBOSA, 2014).

7) Que potencialidades e limitações emergem nos estudos sobre a utilização do modelo TPACK na formação de professores? As potencialidades e limitações do uso do modelo TPACK nos cursos de formação de professores foram elencadas de acordo com os apontamentos dos próprios autores dos 
estudos selecionados nesta revisão. Os temas mais recorrentes são apresentados a seguir:

Potencialidades: o modelo TPACK para formação de professores é um campo de estudos aberto a ser explorado, pois há inúmeras formas de integração de seus domínios com as diferentes áreas do conhecimento [A22], revelando-se um modelo útil para esclarecer conjuntos didáticos [A28] e para orientar a organização e gestão de programas de formação [A2 1].

A possibilidade de refletir sobre a prática e a aplicabilidade das TIC para resolução de problemas [A2], principalmente de forma cooperativa entre os cursistas [A7, A28], implicou na valorização da vertente prática e experimental do modelo TPACK [A10, A25, A26]. Isso conferiu mais segurança para utilização dos recursos tecnológicos [A 1, A 10], rompendo, desta forma, com a visão tecnicista a favor do desenvolvimento de competências mais amplas [A24]. Santos Neto e Struchiner (2019), em sua revisão sistemática sobre o uso do referencial TPACK na formação de professores de ciências, no período de 2006 a 2018, encontrou quatro estudos sobre a avaliação de cursos e estratégias para o desenvolvimento do TPACK dos professores; os resultados, assim como os descritos anteriormente, indicaram que a reflexão e a solução de problemas são os principais elementos promotores do TPACK e devem estar presentes no planejamento de cursos de formação.

Por fim, todos esses fatores potencializaram e ampliaram os conhecimentos prévios dos cursistas sobre a temática $[\mathrm{A} 1]$, preenchendo, desta forma, possíveis lacunas da formação inicial [A26].

Limitações: quanto à formação inicial, a pouca experiência dos futuros professores em sala de aula interferiu no planejamento eficaz de estratégias de ensino com tecnologia integrada [A6, A23, A27]. Alguns estudos sugerem, inclusive, que mesmo os licenciandos considerados "nativos digitais" apresentam conhecimento TCK relativamente fraco, e a Universidade pouco contribui para seu desenvolvimento (SANTOS NETO; STRUCHINER, 2019). Wang, SchmidtCrawford e Jin (2018) por sua vez, indicaram que os domínios centrais básicos como TK, PK e CK são facilmente detectados e podem se desenvolver ao longo da formação inicial, em oposição aos domínios PCK, TCK e TPK, os quais não adquirem avanços ou não são identificados nos estudos com licenciandos.

Na formação continuada, a pouca familiaridade dos cursistas com recursos tecnológicos foi apontada como limitadora para o desenvolvimento 
do conhecimento TPACK como um todo [A 1], e se origina em uma formação inicial menos efetiva e pouco voltada ao letramento informacional e digital $[\mathrm{A} 14, \mathrm{~A} 15, \mathrm{~A} 25, \mathrm{~A} 28]$, trazendo, como resultado, a dificuldade na aquisição e domínio do conhecimento tecnológico (TK) [A22].

Os demais fatores limitantes, comuns a ambos os tipos de formação, foram: a falta de tempo para apropriação, pelos cursistas, de todo o conteúdo de um curso desenhado com base no TPACK [A25, A26, A27], o que exigiria um estudo contínuo e não concentrado em poucos meses; seu alto nível de complexidade e as diferentes visões conceituais dos formadores e cursistas sobre alguns domínios (VOOG; FISSER; PAREJA-ROBLIN; TONDEUR; VAN BRAAK, 20131, o que demandaria negociações de significado de ambos para compreensão global do TPACK [A21]; a resistência a mudanças e insegurança dos cursistas quanto ao uso das tecnologias [A 12, A27], as quais, segundo Maneira e Gomes (2016) em sua revisão sobre a formação continuada de professores em Portugal, podem ser superadas por meio da "compreensão e operacionalização do TPACK pelos professores" (MANEIRA; GOMES, 2016 , p. 1357) e o maior tempo de preparo exigido dos formadores para planejar dinâmicas que contemplem todos os sete domínios do TPACK [A 14, A20].

\section{Considerações Finais}

Esta revisão sistemática teve como objetivo identificar, em estudos do Brasil e em Portugal, de que forma o quadro TPACK é utilizado como modelo teórico para o desenho de cursos de formação de professores (inicial ou continuada). O modelo TPACK mostrou-se uma importante base teórica orientadora dos estudos analisados, contribuindo para a complexa integração das tecnologias com os conhecimentos pedagógicos e de conteúdo específico.

Dentre os resultados desta revisão sistemática, cabe destaque à prevalência de cursos de formação continuada, na área científica da Matemática e com abordagem voltada para o desenvolvimento do domínio TPK dos professores. A baixa incidência de estudos na área da formação inicial vem acompanhada de discussões sobre a falta de incentivo das universidades para uma formação adequada em tecnologias, fazendo com que seus reflexos sejam sentidos no desenvolvimento de uma formação continuada mais efetiva. 
Por fim, foi encontrado apenas um trabalho voltado para formação de professores para atuar em Educação a Distância, indicando um campo de estudos a ser explorado, principalmente devido à crescente oferta de cursos online. Discutir o TPACK no âmbito da docência virtual é fundamental, devido ao uso intenso das tecnologias nesta modalidade, exigindo-se, assim, uma formação constante e específica destes professores.

\section{Referências}

ALMEIDA, Maria Elizabeth Bianconcini de. Educação e tecnologias no Brasil e em Portugal em três momentos de sua história. Educação, Formação \& Tecnologias, Lisboa, v. 1, n. 1, p. 23-36, 2008.

BARBOSA, Guilherme Lopes. Repensar as TIC nas práticas letivas - da formação à integração: um estudo com educadores de infância e professores do $1^{\circ}$ ciclo do Ensino Básico. 2014, 256f. Dissertação (Mestrado em Ciências da Educação) - Instituto de Educação, Universidade do Minho, Braga (Portugall). 2014.

BERNARDES, Thaygra Severo; ANDRADE NETO, Agostinho Serrano de. Technological pedagogical Content Knowledge (TPACK) in pre-service and in-service chemistry teacher training: a systematic literature review. RENOTE, Porto Alegre, v. 18, n. 2, p. 611 1-620, dez. 2020.

BORN, Bárbara Barbosa; PRADO, Ana Pires do; FELIPPE, Janaína Mourão Freire Gori. Profissionalismo docente e estratégias para o seu fortalecimento: entrevista com Lee Shulman. Educação e Pesquisa, São Paulo, v. 45, jan. 2019.

$\mathrm{CHAl}$, Ching Sing; KOH, Joyce Hwee Ling; TSAl, Chin-Chung. A Review of Technological Pedagogical Content Knowledge. Educational Technology \& Society, Douliu, v. 16, n. 2, p. 31-51, abr. 2013.

FERNANDEZ, Carmen. PCK-conhecimento pedagógico do conteúdo: perspectivas e possibilidades para a formação de professores. In: ENCONTRO NACIONAL DE PESQUISA EM EDUCAÇÃO EM CIÊNCIAS, 8., 201 1, Campinas. Anais [...]. Rio de Janeiro: Abrapec, 2011. 1 Online.

GOUGH, David; THOMAS, James; OLIVER, Sandy. Clarifying differences between review designs and methods. Systematic Reviews, Londres, v. 1, n. 1, jun. 2012.

HARRIS, Judi. Inservice teachers' TPACK development: Trends, models, and trajectories. In: HERRING, Mary; KOEHLER, Matthew; MISHRA Punya (ed.). Handbook of technological pedagogical content knowledge for educators. 2. ed. Nova York: Routledge, 2016. 
HERRING, Mary C.; KOEHLER, Matthew J.; MISHRA, Punya (ed.). Handbook of technological pedagogical content knowledge (TPACK) for educators. 2. ed. Nova York: Routledge, 2016.

HOFER, Mark; HARRIS, Judith. TPACK research with inservice teachers: where's the TCK? In: Society for Information Technology \& Teacher Education International Conference, 2012, Austin. Proceedings [...]. Waynesville: Association for the Advancement of Computing in Education, 2012. 1 Online.

KITCHENHAM, Barbara. Procedures for performing systematic reviews. Keele University Technical Report TR/SE-0401. Keele: Keele University, 2004.

MANEIRA, Simone; GOMES, Maria João. Professores e TPACK: uma revisão sistemática da literatura. In: CONGRESSO MUNDIAL ESTILOS DE APRENDIZAGEM, 7., 2016, Braga. Atas [.. ]. Bragança: Instituto Politécnico de Bragança, 2016. 1 Online.

MARINHO, Cíntia Morais. Contribuições do quadro tpack para formação de professores de língua portuguesa: uma proposta de trilha de aprendizagem. 2018, 76f. Dissertação (Mestrado em Educação) - Instituło de Educação, Universidade de Lisboa, Lisboa, 2018.

MISHRA, Punya; KOEHLER, Matthew. Technological pedagogical content knowledge: A framework for teacher knowledge. Teachers College Record, Nova York, v. 108, n. 6, p. 1017-1054, jun. 2006.

MORAN, José. As mídias na educação. In: MORAN, José. Desafios na comunicação pessoal. 3. ed. São Paulo: Paulinas, 2007.

MOUZA, Chrystalla. Developing and assessing TPACK among pre-service teachers. In: HERRING, Mary; KOEHLER, Matthew; MISHRA Punya (ed.). Handbook of technological pedagogical content knowledge for educators. 2. ed. Nova York: Routledge, 2016.

MOUZA, Chrystalla; NANDAKUMAR, Ratna; OZDEN, Sule Yilmaz; KARCHMER-KLEIN, Rachel. A longitudinal examination of preservice teachers' technological pedagogical content knowledge in the context of undergraduate teacher education. Action in Teacher Education, Londres, v. 39, n. 2, p. 153-171, abr. 2017.

NOGUEIRA, Fernando; PESSOA, Teresa; GALLEGO, Maria-Jesus. Desafios e oportunidades do uso da tecnologia para a formação contínua de professores: uma revisão em torno do TPACK em Portugal, Brasil e Espanha. \#Tear: Revista de Educação, Ciência e Tecnologia, Canoas, v. 4, n. 2, dez. 2015.

OLIVEIRA, Hélia; HENRIQUES, Ana; GUTIÉRREZ-FALLAS, Luis Fabián. A integração da tecnologia na planificação de aulas na perspectiva do ensino exploratório: um estudo com futuros professores de matemática. Perspectiva, Florianópolis, v. 36, n. 2, p. 421-446, jun. 2018. 
PERUSKI, Lisa; MISHRA, Punya. Webs of activity in online course design and teaching. Research in Learning Technology, Bicester, v. 12, n. 1, p. 37-49, mar. 2004.

ROLANDO, Luiz Gustavo Ribeiro; LUZ, Maurício Roberto Motta Pinto da; SALVADOR, Daniel Fábio. $\bigcirc$ conhecimento tecnológico pedagógico do conteúdo no contexto lusófono: uma revisão sistemática da literatura. Revista Brasileira de Informática na Educação, Porto Alegre, v. 23, n. 3, p. 174-190, set./dez. 2015.

ROSÁRIO, Lina Sara Chovano do; MOREIRA, António. Competências do professor para o ensino online: análise de um curso de capacitação de docentes em EaD. Indagatio Didactica, Aveiro, v. 7, n. 1, p. 116-131, jan. 2015.

ROSENBERG, Joshua; KOEHLER, Matthew. Context and technological pedagogical content knowledge (TPACK): a systematic review. Journal of Research on Technology in Education, Londres, v. 47, n. 3, p. 186-210, ago. 2015.

SANTOS NETO, Raul dos; STRUCHINER, Miriam. Um panorama sobre a integração do conhecimento tecnológico na formação de professores de ciências. Revista Latinoamericana de Tecnología Educativa, Badajoz, v. 18, n. 2, p. 219-231, 2019.

SHULMAN, Lee. Those who understand: knowledge growth in teaching. Educational Researcher, Washington, v. 15, n. 2, p. 4-14, fev. 1986.

SHULMAN, Lee. Knowledge and teaching: foundations of the new reform. Harvard Educational Review, Cambridge, v. 57, n. 1, p. 1-23, abr. 1987.

SOLER-COSTA, Rebeca; MORENO-GUERRERO, Antonio José; LÓPEZ-BELMONTE, Jesús;MARÍN-MARÍN, José Antonio. Co-Word Analysis and Academic Performance of the Term TPACK in Web of Science. Sustainability, Basileia, v. 13, n. 3, fev. 2021.

VOOGT, Joke; FISSER, Petra; PAREJA ROBLIN, Natalie; TONDEUR, Jo; VAN BRAAK, Johan. Technological pedagogical content knowledge-a review of the literature. Journal of computer assisted learning, Hoboken, v. 29, n. 2, p. 109-121, mar. 2013.

WANG, Wei; SCHMIDT-CRAWFORD, Denise; IIN, Yi. Preservice teachers' TPACK development: a review of literature. Journal of Digital Learning in Teacher Education, Londres, $V$. 34, n. 4, p. 234-258, out. 2018. 
Artigo

Revisão sistemática de estudos sobre TPACK na formação de professores no Brasil e em Portugal

Mestranda Priscilla Ramos Lara Ribeiro

Instituto de Educação da Universidade de Lisboa (Portugal)

Mestrado em Educação e Tecnologias Digitais

Orcid id: https:/ / orcid.org/ 0000-0003-1661-0711

E-mail: priscilla.ribeiro@edu.ulisboa.pt

Prof. Dr. João Manuel Nunes Piedade

Instituto de Educação da Universidade de Lisboa (Portugal)

Unidade de Investigação e Desenvolvimento em Educação e Formação

Orcid id: https: / / orcid.org/0000-0002-4 11 18-397X

E-mail: jmpiedade@ie.ulisboa.pt

Recebido 19 mar. 2021

Aceito 7 maio 2021 